\title{
On the solution of two-dimensional fractional Black-Scholes equation for European put option
}

\author{
Din Prathumwan ${ }^{1}$ (D) and Kamonchat Trachoo ${ }^{2^{*}}$ (D)
}

${ }^{*}$ Correspondence:
kamonchat.t@msu.ac.th
${ }^{2}$ Department of Mathematics,
Faculty of Science, Mahasarakham
University, Mahasarakham, Thailand
Full list of author information is
available at the end of the article

available at the end of the article

\begin{abstract}
The purpose of this paper was to investigate the dynamics of the option pricing in the market through the two-dimensional time fractional-order Black-Scholes equation for a European put option. The Liouville-Caputo derivative was used to improve the ordinary Black-Scholes equation. The analytic solution is a powerful tool for describing the behavior of the option price in the European style market. In this study, analytic solution is carried out by the Laplace homotopy perturbation method. Moreover, the obtained solution showed that the Laplace homotopy perturbation method was an efficient method for finding an analytic solution of two-dimensional fractional-order differential equation.
\end{abstract}

Keywords: Analytical solutions; Fractional Black-Scholes equation; European put option; Laplace homotopy perturbation method

\section{Introduction}

Fractional calculus is widely used to model many real-life problems in various fields such as physics, engineering, biology, earth science, chemistry, finance, and so on [1-8]. The fractional calculus was introduced more than 300 years ago [9]. In the beginning, it was only in a theoretical sense. Recently, fractional calculus was wildly studied in many ways [5, 9-14]. Moreover, the fractional calculus has shown to be more accurate for describing real complicated phenomena than the ordinary calculus $[15,16]$. There are many version of fractional calculus such as Riemann-Liouville [5], Hadamard [17], Atangana-Baleanu [18], Liouville-Caputo [19], Riesz [14], etc.

Actually, one cannot find exact solutions to most fractional-order differential equations, so the approximate solutions are investigated to solve linear and nonlinear fractionalorder differential equations. There are many researches dealing with fractional order differential equations in many different fields [20-23].

The main feature of fractional order differential equations is memory. The variables in financial problems have long memories. Therefore, the fractional order differential equations are fitted to describe the financial problems. Many researches have been done to investigate the fractional-order differential equations in financial problems [24-26].

(c) The Author(s) 2020. This article is licensed under a Creative Commons Attribution 4.0 International License, which permits use, sharing, adaptation, distribution and reproduction in any medium or format, as long as you give appropriate credit to the original author(s) and the source, provide a link to the Creative Commons licence, and indicate if changes were made. The images or other third party material in this article are included in the article's Creative Commons licence, unless indicated otherwise in a credit line to the material. If material is not included in the article's Creative Commons licence and your intended use is not permitted by statutory regulation or exceeds the permitted use, you will need to obtain permission directly from the copyright holder. To view a copy of this licence, visit http://creativecommons.org/licenses/by/4.0/. 
The Black-Scholes equation was proposed by Fisher Black and Myron Scholes in 1973 [27] to express the behavior of the option price in the European style market. Several papers have investigated how the Black-Scholes equation describes the behavior of the market [28-32]. Moreover, the Black-Scholes equation has been extended to express the behavior in another market, such as the American-style market and Asian-style market [33-36].

In general, the two-dimensional Black-Scholes equations for a European put option can be written as follows:

$$
\begin{aligned}
& \frac{\partial P}{\partial \tau}+\frac{1}{2} \sum_{i=1}^{2} \sum_{j=1}^{2} \sigma_{i} \sigma_{j} \rho_{i j} x_{i} x_{j} \frac{\partial^{2} P}{\partial x_{i} \partial x_{j}}+\sum_{i=1}^{2}\left(r-q_{i}\right) x_{i} \frac{\partial P}{\partial x_{i}}-r P=0, \\
& x_{1}, x_{2} \in[0, \infty), \quad \tau \in[0, T]
\end{aligned}
$$

with the terminal condition

$$
P\left(x_{1}, x_{2}, T\right)=\max \left(E-\sum_{i=1}^{2} \tilde{\beta}_{i} x_{i}, 0\right),
$$

where $E=\max \left\{E_{1}, E_{2}\right\}$, and the boundary conditions:

$$
\begin{aligned}
& P\left(x_{1}, x_{2}, \tau\right)=0 \quad \text { as }\left(x_{1}, x_{2}\right) \rightarrow(0,0), \\
& P\left(x_{1}, x_{2}, \tau\right)=\sum_{i=1}^{2} \tilde{\beta}_{i} x_{i}-E e^{-r(T-\tau)} \quad \text { as } x_{1} \rightarrow \infty \text { or } x_{2} \rightarrow \infty
\end{aligned}
$$

where $P$ denotes the value of a put option of the underlying stock prices $\left\{x_{1}, x_{2}\right\}$ at time $\tau, q_{i}$ denotes the dividend yield on the $i$ th underlying stock, $\rho_{i j}$ denotes the correlation between the $i$ th and $j$ th underlying stock prices, $T$ denotes the expiration date, $r$ denotes the risk-free interest rate, $\sigma_{i}$ denotes the volatility of the $i$ th underlying stock, $E_{i}$ denotes the strike price of the $i$ th underlying stock, and $\tilde{\beta}_{i}$ denotes a coefficient so that prices of all the risky assets are at the same level.

There are many researchers who investigated analytical and approximate solutions of the Black-Scholes equation. Various effective methods have been used to solve the BlackScholes equation, for example, the finite difference method [37], finite element method $[15,38]$, homotopy perturbation method [39, 40], the Mellin transform method [41], Adomian decomposition method [42], the variational iteration method [43, 44], radial basis function partition of unity method (RBF-PUM) [45, 46], and adaptive moving mesh method [47].

In this paper, we apply the Laplace homotopy perturbation method (LHPM) to get the analytical solution of the fractional-order Black-Scholes equation. This method is a combination of the Laplace transform and homotopy perturbation method [48]. It provides an analytical solution in the form of a convergent series. From the mathematical point of view, the analytical solution is a useful tool to describe the behavior of the solution, particularly the financial behavior of the solution to fractional-order differential equations.

The paper is structured as follows: the basic knowledge about fractional calculus is introduced in Sect. 2, and the two-dimensional time-fractional Black-Scholes equation is 
presented in Sect. 3. In Sect. 4, the analytical solution of the model is obtained by LHPM, followed by the conclusions in Sect. 5 .

\section{Mathematical background}

\subsection{Fractional calculus}

In this section, we present the definitions of fractional calculus which are used in this paper.

Definition 1 The Riemann-Liouville integral operator of the fractional order $0<\alpha<1$ for a function $f:(0, \infty) \rightarrow \mathbb{R}$ is defined as [5]:

$$
J_{t}^{\alpha} f(t)=\frac{1}{\Gamma(\alpha)} \int_{0}^{t}(t-s)^{\alpha-1} f(s) d s
$$

where $\Gamma$ is the well-known Gamma function.

Riemann-Liouville fractional integral operator has some properties which are stated as follows: for any $\alpha, \beta \geq 0$ and $\gamma>-1$,

$$
\begin{aligned}
& J^{\alpha} J^{\beta} f(t)=J^{\alpha+\beta} f(t), \\
& J^{\alpha} J^{\beta} f(t)=J^{\beta} J^{\alpha} f(t), \\
& J^{\alpha} t^{\beta}=\frac{\Gamma(\gamma+1}{\Gamma(\gamma+\alpha+1)} t^{\alpha+\beta} .
\end{aligned}
$$

Definition 2 The Riemann-Liouville derivative of the fractional order $0<\alpha<1$ for a function $f:(0, \infty) \rightarrow \mathbb{R}$ is defined as [5]:

$$
{ }^{\mathrm{RL}} D_{t}^{\alpha} f(t)=\frac{1}{\Gamma(1-\alpha)} \frac{d}{d t} \int_{0}^{t} \frac{f(s)}{(t-s)^{\alpha}} d s .
$$

Definition 3 The Liouville-Caputo-type fractional derivative of order $0<\alpha \leq 1$ for a function $f:(0, \infty) \rightarrow \mathbb{R}$ is defined as [19]:

$$
D_{\tau}^{\alpha} f(\tau)= \begin{cases}\frac{1}{\Gamma(1-\alpha)} \int_{0}^{\tau} \frac{f^{\prime}(s)}{(\tau-\theta)^{\alpha}} d \theta, & 0<\alpha<1, \\ \frac{d f}{d \tau}, & \alpha=1 .\end{cases}
$$

In this paper, we use Liouville-Caputo derivative as the time-fractional derivative because the initial condition for the fractional order derivation is similar to the traditional derivative [49].

Definition 4 The Mittag-Leffler function is defined as [50]:

$$
E_{\alpha}(z)=\sum_{k=0}^{\infty} \frac{z^{k}}{\Gamma(\alpha k+1)}, \quad \alpha \in \mathbb{C}, \operatorname{Re}(\alpha)>0
$$

Definition 5 The generalized Mittag-Leffler function is defined as [50]:

$$
E_{\alpha, \beta}(z)=\sum_{k=0}^{\infty} \frac{z^{k}}{\Gamma(\alpha k+\beta)}, \quad \alpha, \beta \in \mathbb{C}, \operatorname{Re}(\alpha)>0, \operatorname{Re}(\beta)>0 .
$$




\section{Generalized time fractional-order Black-Scholes model}

The standard two-dimensional Black-Scholes equation for a European put option was presented in Sect. 1. Throughout this paper, we assume that $\sigma_{1}, \sigma_{2}, \rho$, and $r$ are constants.

By transforming coordinates and time to a forward time coordinate $t=T-\tau$ and applying the Liouville-Caputo fractional derivative to the two-dimensional Black-Scholes equation, we obtain the following two-dimension time-fractional order Black-Scholes equation with $\alpha \in(0,1]$ and the initial and boundary conditions as follows:

$$
D_{t}^{\alpha} \omega=\frac{1}{2} \sigma_{1}^{2} \frac{\partial^{2} \omega}{\partial x^{2}}+\frac{1}{2} \sigma_{2}^{2} \frac{\partial^{2} \omega}{\partial y^{2}}+\rho \sigma_{1} \sigma_{2} \frac{\partial^{2} \omega}{\partial x \partial y}, \quad(x, y, t) \in \mathbb{R} \times \mathbb{R} \times[0, T],
$$

subject to the initial condition:

$$
\omega(x, y, 0)=\max \left(E-\left(\beta_{1} e^{x}+\beta_{2} e^{y}\right), 0\right),
$$

and the boundary conditions:

$$
\begin{aligned}
& \omega(x, y, t)=0 \quad \text { as }(x, y) \rightarrow-\infty, \quad \text { and } \\
& \omega(x, y, t)=E-\left(\beta_{1} e^{x+\frac{1}{2} \sigma_{1}^{2} t}+\beta_{2} e^{y+\frac{1}{2} \sigma_{2}^{2} t}\right) \quad \text { as } x \rightarrow \infty \text { or } y \rightarrow \infty,
\end{aligned}
$$

where $\beta_{1}=\tilde{\beta}_{1} e^{\left(r-\frac{1}{2} \sigma_{1}^{2}\right) T}$ and $\beta_{2}=\tilde{\beta}_{2} e^{\left(r-\frac{1}{2} \sigma_{2}^{2}\right) T}$.

\section{Solving two-dimensional time-fractional Black-Scholes equation by LHPM}

In this section, we apply LHPM techniques for finding the solution of two-dimensional time-fractional Black-Scholes model (5) subject to the initial condition (6) and the boundary conditions (7).

Theorem 1 The analytical solution of two-dimensional time-fractional Black-Scholes model (5) is given by

$$
\begin{aligned}
\omega(x, y, t)= & \max \left(E-\left(\beta_{1} e^{x}+\beta_{2} e^{y}\right), 0\right)+e^{x+y} t^{\alpha} \\
& +\max \left(\beta_{1} e^{x}, 0\right) \frac{t^{\alpha} \sigma_{1}^{2}}{2} E_{\alpha, \alpha+1}\left(\frac{t^{\alpha} \sigma_{1}^{2}}{2}\right)+\max \left(\beta_{2} e^{y}, 0\right) \frac{t^{\alpha} \sigma_{2}^{2}}{2} E_{\alpha, \alpha+1}\left(\frac{t^{\alpha} \sigma_{2}^{2}}{2}\right) \\
& +\left[e^{x+y}\left(\frac{\sigma_{1}^{2}}{2}+\frac{\sigma_{2}^{2}}{2}+\rho \sigma_{1} \sigma_{2}\right) \Gamma(\alpha+1) t^{2 \alpha} E_{\alpha, 2 \alpha+1}\left(t^{\alpha}\left(\frac{\sigma_{1}^{2}}{2}+\frac{\sigma_{2}^{2}}{2}+\rho \sigma_{1} \sigma_{2}\right)\right)\right] \\
& -e^{x+y} \Gamma(\alpha+1) t^{\alpha} E_{\alpha, \alpha+1}\left(t^{\alpha}\left(\frac{\sigma_{1}^{2}}{2}+\frac{\sigma_{2}^{2}}{2}+\rho \sigma_{1} \sigma_{2}\right)\right),
\end{aligned}
$$

where $E_{\gamma, \eta}(z)=\sum_{k=0}^{\infty} \frac{z^{k}}{\Gamma(\gamma k+\eta)}$ is the generalized Mittag-Leffler function, in which $\gamma$ and $\eta$ are constants.

Proof Let

$$
\tilde{N}(\omega(x, y, t))=\frac{1}{2} \sigma_{1}^{2} \frac{\partial^{2} \omega}{\partial x^{2}}+\frac{1}{2} \sigma_{2}^{2} \frac{\partial^{2} \omega}{\partial y^{2}}+\rho \sigma_{1} \sigma_{2} \frac{\partial^{2} \omega}{\partial x \partial y},
$$


and represent Eq. (5) in the general form of

$$
D_{t}^{\alpha} \omega(x, y, t)=\tilde{N}(\omega(x, y, t)) .
$$

Applying the Laplace transform with respect to $t$, we obtain

$$
\mathcal{L}\left\{D_{t}^{\alpha} \omega(x, y, t)\right\}=\mathscr{L}\{\tilde{N}(\omega(x, y, t))\} .
$$

By the properties of Laplace transform of the Liouville-Caputo fractional derivative [51], Eq. (8) becomes

$$
\begin{aligned}
\mathcal{L}\{\omega(x, y, t)\}= & \frac{1}{s} \max \left(E-\left(\beta_{1} e^{x}+\beta_{2} e^{y}\right), 0\right) \\
& +\frac{1}{s^{\alpha}} \mathcal{L}\left\{\frac{1}{2} \sigma_{1}^{2} \frac{\partial^{2} \omega}{\partial x^{2}}+\frac{1}{2} \sigma_{2}^{2} \frac{\partial^{2} \omega}{\partial y^{2}}+\rho \sigma_{1} \sigma_{2} \frac{\partial^{2} \omega}{\partial x \partial y}\right\} .
\end{aligned}
$$

Taking the inverse Laplace transform of (9), we get

$$
\begin{aligned}
\omega(x, y, t)= & \max \left(E-\left(\beta_{1} e^{x}+\beta_{2} e^{y}\right), 0\right) \\
& +\mathcal{L}^{-1}\left\{\frac{1}{s^{\alpha}} \mathcal{L}\left\{\frac{1}{2} \sigma_{1}^{2} \frac{\partial^{2} \omega}{\partial x^{2}}+\frac{1}{2} \sigma_{2}^{2} \frac{\partial^{2} \omega}{\partial y^{2}}+\rho \sigma_{1} \sigma_{2} \frac{\partial^{2} \omega}{\partial x \partial y}\right\}\right\} .
\end{aligned}
$$

Applying techniques of HPM, function $\omega$ can be constructed

$$
\omega(x, y, t ; q): \mathbb{R} \times \mathbb{R} \times[0, T] \times[0,1] \rightarrow \mathbb{R}
$$

which satisfies the following equation:

$$
\begin{aligned}
& (1-q)\left(\omega(x, y, t ; q)-\widetilde{\omega}_{0}(x, y, t)\right)+q\left[\omega(x, y, t ; q)-\max \left(E-\left(\beta_{1} e^{x}+\beta_{2} e^{y}\right), 0\right)\right. \\
& \left.-\mathcal{L}^{-1}\left\{\frac{1}{s^{\alpha}} \mathcal{L}\left\{\frac{1}{2} \sigma_{1}^{2} \frac{\partial^{2} \omega}{\partial x^{2}}+\frac{1}{2} \sigma_{2}^{2} \frac{\partial^{2} \omega}{\partial y^{2}}+\rho \sigma_{1} \sigma_{2} \frac{\partial^{2} \omega}{\partial x \partial y}\right\}\right\}\right]=0,
\end{aligned}
$$

or

$$
\begin{aligned}
\omega(x, y, t ; q)= & \widetilde{\omega}_{0}(x, y, t)-q \widetilde{\omega}_{0}(x, y, t)+q \max \left(E-\left(\beta_{1} e^{x}+\beta_{2} e^{y}\right), 0\right) \\
& +q \mathcal{L}^{-1}\left\{\frac{1}{s^{\alpha}} \mathcal{L}\left\{\frac{1}{2} \sigma_{1}^{2} \frac{\partial^{2} \omega}{\partial x^{2}}+\frac{1}{2} \sigma_{2}^{2} \frac{\partial^{2} \omega}{\partial y^{2}}+\rho \sigma_{1} \sigma_{2} \frac{\partial^{2} \omega}{\partial x \partial y}\right\}\right\},
\end{aligned}
$$

where $q \in[0,1]$ is an embedded parameter and $\widetilde{\omega}_{0}(x, y, t)$ is an initial approximation of Eq. (10) which can freely be chosen [52]. For this case, we choose $\widetilde{\omega}_{0}(x, y, t)$ as

$$
\widetilde{\omega}_{0}(x, y, t)=\max \left(E-\left(\beta_{1} e^{x}+\beta_{2} e^{y}\right), 0\right)+e^{x+y} t^{\alpha} .
$$

Then, we substitute $\widetilde{\omega}_{0}(x, y, t)$ into Eq. $(10)$ to get

$$
\begin{aligned}
\omega(x, y, t ; q)= & \max \left(E-\left(\beta_{1} e^{x}+\beta_{2} e^{y}\right), 0\right)+e^{x+y} t^{\alpha} \\
& +q\left(-e^{x+y} t^{\alpha}+\mathcal{L}^{-1}\left\{\frac{1}{s^{\alpha}} \mathcal{L}\left\{\frac{1}{2} \sigma_{1}^{2} \frac{\partial^{2} \omega}{\partial x^{2}}+\frac{1}{2} \sigma_{2}^{2} \frac{\partial^{2} \omega}{\partial y^{2}}+\rho \sigma_{1} \sigma_{2} \frac{\partial^{2} \omega}{\partial x \partial y}\right\}\right\}\right) .
\end{aligned}
$$


For HPM, the solution of Eq. (5) can be assumed to be of the form

$$
\omega(x, y, t ; q)=\sum_{i=0}^{\infty} q^{n} \phi_{n}(x, y, t)
$$

Substituting Eq. (12) into Eq. (11), Eq. (11) becomes

$$
\begin{aligned}
\sum_{n=0}^{\infty} q^{n} \phi_{n}(x, y, t)= & \max \left(E-\left(\beta_{1} e^{x}+\beta_{2} e^{y}\right), 0\right)+e^{x+y} t^{\alpha} \\
& +q\left(-e^{x+y} t^{\alpha}+\mathcal{L}^{-1}\left\{\frac { 1 } { s ^ { \alpha } } \mathcal { L } \left\{\frac{1}{2} \sigma_{1}^{2} \sum_{n=0}^{\infty} q^{n} \frac{\partial^{2} \phi_{n}}{\partial x^{2}}\right.\right.\right. \\
& \left.\left.\left.+\frac{1}{2} \sigma_{2}^{2} \sum_{n=0}^{\infty} q^{n} \frac{\partial^{2} \phi_{n}}{\partial y^{2}}+\rho \sigma_{1} \sigma_{2} \sum_{n=0}^{\infty} q^{n} \frac{\partial^{2} \phi_{n}}{\partial x \partial y}\right\}\right\}\right) .
\end{aligned}
$$

When we equate the corresponding powers of $q$ of Eq. (13), we have

$$
\begin{aligned}
\phi_{0}(x, y, t)= & \max \left(E-\left(\beta_{1} e^{x}+\beta_{2} e^{y}\right), 0\right)+e^{x+y} t^{\alpha}, \\
\phi_{1}(x, y, t)= & -e^{x+y} t^{\alpha}+\mathcal{L}^{-1}\left\{\frac{1}{s^{\alpha}} \mathcal{L}\left\{\frac{1}{2} \sigma_{1}^{2} \frac{\partial^{2} \phi_{0}}{\partial x^{2}}+\frac{1}{2} \sigma_{2}^{2} \frac{\partial^{2} \phi_{0}}{\partial y^{2}}+\rho \sigma_{1} \sigma_{2} \frac{\partial^{2} \phi_{0}}{\partial x \partial y}\right\}\right\}, \\
\phi_{i}(x, y, t)= & -e^{x+y} t^{\alpha} \\
& +\mathcal{L}^{-1}\left\{\frac{1}{s^{\alpha}} \mathcal{L}\left\{\frac{1}{2} \sigma_{1}^{2} \frac{\partial^{2} \phi_{i-1}}{\partial x^{2}}+\frac{1}{2} \sigma_{2}^{2} \frac{\partial^{2} \phi_{i-1}}{\partial y^{2}}+\rho \sigma_{1} \sigma_{2} \frac{\partial^{2} \phi_{i-1}}{\partial x \partial y}\right\}\right\} \quad \text { for } i \geq 2 .
\end{aligned}
$$

Then, we can write $\phi_{0}, \phi_{1}, \phi_{2}, \phi_{3}, \ldots$ in the general form, i.e.,

$$
\begin{aligned}
\phi_{0}(x, y, t)= & \max \left(E-\left(\beta_{1} e^{x}+\beta_{2} e^{y}\right), 0\right)+e^{x+y} t^{\alpha} \\
\phi_{n}(x, y, t)= & \frac{t^{n \alpha}}{\Gamma(n \alpha+1)}\left(\frac{1}{2^{n}} \sigma_{1}^{2 n} \max \left(\tilde{\beta}_{1} e^{x}, 0\right)+\frac{1}{2^{n}} \sigma_{2}^{2 n} \max \left(\tilde{\beta}_{2} e^{y}, 0\right)\right) \\
& +e^{x+y} \frac{t^{(n+1) \alpha} \Gamma(\alpha+1)}{\Gamma((n+1) \alpha+1)}\left(\frac{\sigma_{1}^{2}}{2}+\frac{\sigma_{2}^{2}}{2}+\rho \sigma_{1} \sigma_{2}\right)^{n} \\
& -e^{x+y} \frac{t^{n \alpha} \Gamma(\alpha+1)}{\Gamma(n \alpha+1)}\left(\frac{\sigma_{1}^{2}}{2}+\frac{\sigma_{2}^{2}}{2}+\rho \sigma_{1} \sigma_{2}\right)^{(n-1)} \quad \text { when } n \geq 1 .
\end{aligned}
$$

From Eq. (12), the solution $\omega(x, y, t)$ of the fractional order Black-Scholes equation (5) can be written as

$$
\begin{aligned}
\omega(x, y, t ; q)= & \max \left(E-\left(\beta_{1} e^{x}+\beta_{2} e^{y}\right), 0\right)+e^{x+y} t^{\alpha} \\
& +\sum_{n=0}^{\infty} q^{n+1}\left\{\frac { t ^ { ( n + 1 ) \alpha } } { \Gamma ( ( n + 1 ) \alpha + 1 ) } \left(\frac{1}{2^{(n+1)}} \sigma_{1}^{2(n+1)} \max \left(\beta_{1} e^{x}, 0\right)\right.\right. \\
& \left.+\frac{1}{2^{(n+1)}} \sigma_{2}^{2(n+1)} \max \left(\beta_{2} e^{y}, 0\right)\right) \\
& +e^{x+y} \frac{t^{(n+2) \alpha} \Gamma(\alpha+1)}{\Gamma((n+2) \alpha+1)}\left(\frac{\sigma_{1}^{2}}{2}+\frac{\sigma_{2}^{2}}{2}+\rho \sigma_{1} \sigma_{2}\right)^{(n+1)} \\
& \left.-e^{x+y} \frac{t^{(n+1) \alpha} \Gamma(\alpha+1)}{\Gamma((n+1) \alpha+1)}\left(\frac{\sigma_{1}^{2}}{2}+\frac{\sigma_{2}^{2}}{2}+\rho \sigma_{1} \sigma_{2}\right)^{n}\right\}
\end{aligned}
$$


The solution can be obtained by letting $q \rightarrow 1$, and then we have

$$
\begin{aligned}
\omega(x, y, t ; 1)= & \max \left(E-\left(\beta_{1} e^{x}+\beta_{2} e^{y}\right), 0\right)+e^{x+y} t^{\alpha}+\sum_{n=0}^{\infty}\left\{\frac{t^{(n+1) \alpha}}{\Gamma((n+1) \alpha+1)}\right. \\
& \times\left(\frac{1}{2^{(n+1)}} \sigma_{1}^{2(n+1)} \max \left(\beta_{1} e^{x}, 0\right)+\frac{1}{2^{(n+1)}} \sigma_{2}^{2(n+1)} \max \left(\beta_{2} e^{y}, 0\right)\right) \\
& +e^{x+y} \frac{t^{(n+2) \alpha} \Gamma(\alpha+1)}{\Gamma((n+2) \alpha+1)}\left(\frac{\sigma_{1}^{2}}{2}+\frac{\sigma_{2}^{2}}{2}+\rho \sigma_{1} \sigma_{2}\right)^{(n+1)} \\
& \left.-e^{x+y} \frac{t^{(n+1) \alpha} \Gamma(\alpha+1)}{\Gamma((n+1) \alpha+1)}\left(\frac{\sigma_{1}^{2}}{2}+\frac{\sigma_{2}^{2}}{2}+\rho \sigma_{1} \sigma_{2}\right)^{n}\right\} .
\end{aligned}
$$

Therefore, the solution of Eq. (5) will be

$$
\begin{aligned}
\omega(x, y, t)= & \max \left(E-\left(\beta_{1} e^{x}+\beta_{2} e^{y}\right), 0\right)+e^{x+y} t^{\alpha} \\
& +\max \left(\beta_{1} e^{x}, 0\right) \frac{t^{\alpha} \sigma_{1}^{2}}{2} E_{\alpha, \alpha+1}\left(\frac{t^{\alpha} \sigma_{1}^{2}}{2}\right)+\max \left(\beta_{2} e^{y}, 0\right) \frac{t^{\alpha} \sigma_{2}^{2}}{2} E_{\alpha, \alpha+1}\left(\frac{t^{\alpha} \sigma_{2}^{2}}{2}\right) \\
& +\left[e^{x+y}\left(\frac{\sigma_{1}^{2}}{2}+\frac{\sigma_{2}^{2}}{2}+\rho \sigma_{1} \sigma_{2}\right) \Gamma(\alpha+1) t^{2 \alpha} E_{\alpha, 2 \alpha+1}\left(t^{\alpha}\left(\frac{\sigma_{1}^{2}}{2}+\frac{\sigma_{2}^{2}}{2}+\rho \sigma_{1} \sigma_{2}\right)\right)\right] \\
& -e^{x+y} \Gamma(\alpha+1) t^{\alpha} E_{\alpha, \alpha+1}\left(t^{\alpha}\left(\frac{\sigma_{1}^{2}}{2}+\frac{\sigma_{2}^{2}}{2}+\rho \sigma_{1} \sigma_{2}\right)\right)
\end{aligned}
$$

where $E_{\gamma, \eta}(z)=\sum_{k=0}^{\infty} \frac{z^{k}}{\Gamma(\gamma k+\eta)}$ is the generalized Mittag-Leffler function, in which $\gamma$ and $\eta$ are constants.

\section{Conclusions}

In this paper, we have found the analytical solution of the two-dimensional time-fractional Black-Scholes equation for a European put option using the Liouville-Caputo derivative. The Laplace homotopy perturbation method has been used to obtain the solution which can be written in the form of the generalized Mittag-Leffer function. The main feature of the analytical solution is that it is convenient for finding the option price.

\section{Acknowledgements}

This research was financially supported by Faculty of Science, Mahasarakham University (Grant year 2020).

\section{Funding}

This research received no external funding.

Availability of data and materials

Not applicable.

\section{Competing interests}

The authors declare that they have no competing interests.

\section{Authors' contributions}

All authors contributed equally to this work. All authors read and approved the final manuscript.

\section{Author details}

'Department of Mathematics, Faculty of Science, Khon Kaen University, Khon Kaen, Thailand. ${ }^{2}$ Department of Mathematics, Faculty of Science, Mahasarakham University, Mahasarakham, Thailand. 


\section{Publisher's Note}

Springer Nature remains neutral with regard to jurisdictional claims in published maps and institutional affiliations.

\section{Received: 7 October 2019 Accepted: 18 February 2020 Published online: 03 April 2020}

\section{References}

1. Rekhviashvili, S., Pskhu, A., Agarwal, P., Jain, S.: Application of the fractional oscillator model to describe damped vibrations. Turk. J. Phys. 43(3), 236-242 (2019). https://doi.org/10.3906/fiz-1811-16

2. Tariboon, J., Ntouyas, S.K., Agarwal, P.: New concepts of fractional quantum calculus and applications to impulsive fractional q-difference equations. Adv. Differ. Equ. 2015, Article ID 18 (2015). https://doi.org/10.1186/s13662-014-0348-8

3. Agarwal, P., Choi, J., Bruce, R.: Extended Riemann-Liouville fractional derivative operator and its applications. J. Nonlinear Sci. Appl. 8(5), 451-466 (2015). https://doi.org/10.22436/jnsa.008.05.01

4. Sofuoglu, Y., Ozalp, N.: Fractional order bilingualism model without conversion from dominant unilingual group to bilingual group. Differ. Equ. Dyn. Syst. 25, 1-9 (2017). https://doi.org/10.1007/s12591-015-0239-9

5. Podlubny, l.: Fractional Differential Equations. Mathematics in Science and Engineering. Academic Press, San Diego (1999)

6. Hilfer, R.: Applications of Fractional Calculus in Physics. World Scientific, Hackensack (2001)

7. Mainardi, F.: Fractional relaxation-oscillation and fractional diffusion-wave phenomena. Chaos Solitons Fractals 7(9), 1461-1477 (1996). https://doi.org/10.1016/j.chaos.2017.04.025

8. Agarwal, P., Jain, S., Mansour, T.: Further extended Caputo fractional derivative operator and its applications. Russ. J. Math. Phys. 24(4), 415-425 (2017). https://doi.org/10.1134/S106192081704001X

9. Baleanu, D., Fernandez, A.: On fractional operators and their classifications. Mathematics 7(9), Article ID 830 (2019). https://doi.org/10.3390/math7090830

10. Ruzhansky, M., Cho, Y.J., Agarwal, P., Area, l.: Advances in Real and Complex Analysis with Applications. Birkhäuser, Basel (2017)

11. Agarwal, P.: Further results on fractional calculus of Saigo operators. Appl. Appl. Math. 7(2), 585-594 (2012)

12. Agarwal, P., Al-Mdallal, Q., Cho, Y.J., Jain, S.: Fractional differential equations for the generalized Mittag-Leffler function. Adv. Differ. Equ. 2018, Article ID 58 (2018). https://doi.org/10.1186/s13662-018-1500-7

13. Agarwal, P., Jain, S.: Further results on fractional calculus of Srivastava polynomials. Bull. Math. Anal. Appl. 3(2), 167-174 (2011)

14. Bayin, S.S.: Definition of the Riesz derivative and its application to space fractional quantum mechanics. J. Math. Phys. 57, Article ID 123501 (2016). https://doi.org/10.1063/1.4968819

15. Phaochoo, P., Luadsong, A., Aschariyaphotha, N.: The meshless local Petrov-Galerkin based on moving kriging interpolation for solving fractional Black-Scholes model. J. King Saud Univ., Sci. 28(1), 111-117 (2016). https://doi.org/10.1016/j.jksus.2015.08.004

16. Bjork, T., Hult, H.: A note on Wick products and the fractional Black-Scholes model. Finance Stoch. 9(2), 197-209 (2005). https://doi.org/10.1007/s00780-004-0144-5

17. Hadamard, J.: Implied volatility in Black-Scholes model with GARCH volatility. J. Math. Pures Appl. 4(8), 101-186 (1892)

18. Abdon, A., Dumitru, B.: New fractional derivatives with nonlocal and non-singular kernel: theory and application to heat transfer model. Therm. Sci. 20(2), 763-769 (2016). https://doi.org/10.2298/TSCI160111018A

19. Caputo, M.: Linear models of dissipation whose $q$ is almost frequency independent-II. J. Funct. Spaces 13(5), 529-539 (1967). https://doi.org/10.1111/j.1365-246X.1967.tb02303.x

20. Morales-Delgado, V.F., Gómez-Aguilar, J.F., Saad, K.M., Khan, M.A., Agarwal, P.: Analytic solution for oxygen diffusion from capillary to tissues involving external force effects: a fractional calculus approach. Phys. A, Stat. Mech. Appl. 523, 48-65 (2019). https://doi.org/10.1016/j.physa.2019.02.018

21. Jain, S., Agarwal, P., Kilicman, A.: Pathway fractional integral operator associated with $3 m$-parametric Mittag-Leffler functions. Int. J. Appl. Comput. Math. 4(5), Article ID 115 (2018). https://doi.org/10.1007/s40819-018-0549-z

22. Alderremy, A.A., Saad, K.M., Agarwal, P., Aly, S., Jain, S.: Certain new models of the multi space-fractional Gardner equation. Phys. A, Stat. Mech. Appl. (2019). https://doi.org/10.1016/j.physa.2019.123806

23. Jain, S., Agarwal, P., KIymaz, I.O., Çetinkaya, A.: Some composition formulae for the MSM fractional integral operator with the multi-index Mittag-Leffler functions. AIP Conf. Proc. 1926, Article ID 020020 (2018). https://doi.org/10.1063/1.5020469

24. Sweilam, N.H., Hasan, M.M.A., Baleanu, D.: New studies for general fractional financial models of awareness and trial advertising decisions. Chaos Solitons Fractals 104, 772-784 (2017). https://doi.org/10.1016/j.chaos.2017.09.013

25. Hajipour, A., Hajipour, M., Baleanu, D.: On the adaptive sliding mode controller for a hyperchaotic fractional-order financial system. Phys. A, Stat. Mech. Appl. 497, 139-153 (2018). https://doi.org/10.1016/j.physa.2018.01.019

26. Jajarmi, A., Hajipour, M., Baleanu, D.: New aspects of the adaptive synchronization and hyperchaos suppression of a financial model. Chaos Solitons Fractals 99(9), 285-296 (2017). https://doi.org/10.1016/j.chaos.2017.04.025

27. Black, F., Scholes, M.: The pricing of options and corporate liabilities. J. Polit. Econ. 81(3), 637-654 (1973). https://doi.org/10.1086/260062

28. Osborne, M.F.M.: Brownian motion in the stock market. Oper. Res. 7(2), 145-173 (1959)

29. Kleinert, H., Korbel, J.: Option pricing beyond Black-Scholes based on double-fractional diffusion. Phys. A, Stat. Mech. Appl. 449, 200-214 (2016). https://doi.org/10.1016/j.physa.2015.12.125

30. Al-Zhoura, Z., Barfeieb, M., Soleymanic, F., Tohidi, E.: A computational method to price with transaction costs under the nonlinear Black-Scholes model. Chaos Solitons Fractals 127, 291-301 (2019). https://doi.org/10.1016/j.chaos.2019.06.033

31. David, C.: Control of the Black-Scholes equation. Comput. Math. Appl. 73(7), 1566-1585 (2017). https://doi.org/10.1016/j.camwa.2017.02.007

32. Sheraza, M., Predaab, V.: Implied volatility in Black-Scholes model with GARCH volatility. Proc. Econ. Finance 8 658-663 (2014). https://doi.org/10.1016/S2212-5671(14)00141-5 
33. Alghalith, M.: Pricing the American options using the Black-Scholes pricing formula. Phys. A, Stat. Mech. Appl. 507, 443-445 (2018). https://doi.org/10.1016/..physa.2018.05.087

34. Geske, R., Roll, R.: On valuing American call options with the Black-Scholes European formula. J. Finance 39(2), 443-455 (1984). https://doi.org/10.2307/2327870

35. Vecer, J:: Black-Scholes representation for Asian options. Math. Finance 24(3), 598-626 (2012). https://doi.org/10.1111/mafi.12012

36. Pirjol, D., Zhu, L.: Sensitivities of Asian options in the Black-Scholes model. Int. J. Theor. Appl. Finance 21(1), Article ID 1850008 (2018). https://doi.org/10.1142/S0219024918500085

37. Lesmana, D.C., Wang, S.: An upwind finite difference method for a nonlinear Black-Scholes equation governing European option valuation under transaction costs. J. Appl. Math. Comput. 219(16), 8811-8828 (2013). https://doi.org/10.1016/j.amc.2012.12.077

38. Liu, S., Zhou, Y., Wu, Y., Ge, X.: Option pricing under the jump diffusion and multifactor stochastic processes. J. Funct. Spaces 2019, Article ID 9754679 (2019). https://doi.org/10.1155/2019/9754679

39. Prathumwan, D., Trachoo, K.: Application of the Laplace homotopy perturbation method to the Black-Scholes model based on a European put option with two assets. Mathematics 7(4), Article ID 310 (2019). https://doi.org/10.3390/math7040310

40. Fall, A.N., Ndiaye, S.N., Sene, N.: Black-Scholes option pricing equations described by the Caputo generalized fractional derivative. Chaos Solitons Fractals 125, 108-118 (2019). https://doi.org/10.1016/j.chaos.2019.05.024

41. Yoon, J.H.: Mellin transform method for European option pricing with Hull-White stochastic interest rate. J. Appl. Math. 2014, Article ID 759562 (2014). https://doi.org/10.1155/2014/759562

42. González-Gaxiola, O., de Chávez, J.R., Santiago, J.A.: A nonlinear option pricing model through the Adomian decomposition method. Int. J. Appl. Comput. Math. 2(4), 453-467 (2016). https://doi.org/10.1007/s40819-015-0070-6

43. Safari, M., Ganji, D.D., Moslemi, M.: Application of He's variational iteration method and Adomian's decomposition method to the fractional KdV-Burgers-Kuramoto equation. Comput. Math. Appl. 58(11-12), 2091-2097 (2009). https://doi.org/10.1016/j.camwa.2009.03.043

44. He, J.H.: A short remark on fractional variational iteration method. Phys. Lett. A 375(38), 3362-3364 (2011). https://doi.org/10.1016/j.physleta.2011.07.033

45. Shcherbakov, V., Larsson, E.: Radial basis function partition of unity methods for pricing vanilla basket options. Comput. Math. Appl. 71(1), 185-200 (2016). https://doi.org/10.1016/j.camwa.2015.11.007

46. Mollapourasl, R., Fereshtian, A., Vanmaele, M.: Radial basis functions with partition of unity method for American options with stochastic volatility. Comput. Econ. 53(1), 259-287 (2019). https://doi.org/10.1007/s10614-017-9739-8

47. Huang, J., Cen, Z., Zhao, J.: An adaptive moving mesh method for a time-fractional Black-Scholes equation. Adv. Differ. Equ. 2019. Article ID 516 (2019). https://doi.org/10.1186/s13662-019-2453-1

48. Madani, M., Fathizadeh, M., Khan, Y., Yildirim, A.: On the coupling of the homotopy perturbation method and Laplace transformation. Finance Stoch. 53(9-10), 1937-1945 (2011). https://doi.org/10.1016/j.mcm.2011.01.023

49. Kumar, S., Kumar, D., Singh, J.: Numerical computation of fractional Black-Scholes equation arising in financial market. Egypt. J. Basic Appl. Sci. 1(3-4), 177-183 (2014). https://doi.org/10.1016/j.ejbas.2014.10.003

50. Mathai, A.M., Haubold, H.J.: Special Functions for Applied Scientists. Springer, New York (2008)

51. Miller, K.S., Ross, B.: An Introduction Fractional Calculus Functional Differential Equations. Willey, New York (2003)

52. Baholian, E., Azizi, A., Saeidian, J.: Some notes on using the homotopy perturbation method for solving time-dependent differential equations. Math. Comput. Model. 50(1-2), 213-224 (2009). https://doi.org/10.1016/j.mcm.2009.03.003

\section{Submit your manuscript to a SpringerOpen ${ }^{\circ}$ journal and benefit from:}

- Convenient online submission

- Rigorous peer review

- Open access: articles freely available online

- High visibility within the field

Retaining the copyright to your article

Submit your next manuscript at $\gg$ springeropen.com 